\title{
Finger Vein Verification System based on Three Methodologies of Feature Extraction
}

\author{
Abbas H. Hassin Alasadi \\ Department of Computer Information System, \\ College of Information Technology, \\ Basra University, Iraq.
}

\begin{abstract}
As a new manner of biometrics measurement, human finger vein pattern has been developed. Many researchers have paid close attention to this topic. In this paper, three methodologies of features extraction are used for finger vein verification system. These methods are; Grey Level Co-occurrence Matrix (GLCM), Tamura, and Scale Invariant Feature Transform (SIFT). Empirically, the results of the proposed algorithm was acceptable and better.
\end{abstract}

\section{General Terms}

Biometrics, Pattern Recognition, and Image Processing.

\section{Keywords}

Finger vein, Feature extraction, GLCM, Tamura, SIFT, Matching Algorithm.

\section{INTRODUCTION}

Nowadays, many approaches, such as iris, face, fingerprints, veins patterns, hand geometry, voice, retina, handwriting recognition have been implemented as biometrics authenticate identity system. These biometric techniques are applied in different fields such as crime detection, banking security, physical access control, Information Security, National ID system [1]. Biometric authentication systems have many advantages over a password, smart card, and token. The biometric technique cannot be stolen, forgotten, borrowed or leave at home. This technique is also reliable, efficient and secure. Temperature and diseases may affect the image contrast in this method [2], [3].

Human finger vein pattern, as a new technology, has been developed. Many researchers have paid close attention to this subject, making some of them published many research papers in this field [4], [5], [6]

In comparison with other biometrics, finger vein systems show some prominent advantages in their application. First, finger veins are difficult to forge. This is attributed to the hidden vein patterns being under the skin surface, which makes vein pattern duplication impossible in practice. Secondly, the quality of the captured vein is not easily affected by skin conditions. Furthermore, finger vein patterns are stable and clearly defined, thus allowing a user to use a low-cost and uncomplicated image acquisition device. Finally, in terms of hygiene, finger vein recognition is non-invasive and utilizes contactless imaging, which ensures both suitability and neatness.

Finger vein recognition system captures the data by Near Infrared (NIR) camera. The infrared radiation falls onto the finger. The hemoglobin in the vessels reflexes the dark pattern in the digital images. Images features extraction method should be affected to increase the system efficiency. Images

\author{
Zainab N. Nemer \\ Department of Computer Science, College of \\ Information Technology, \\ Basra University, Iraq
}

are segmented at the different resolution to reduce the error ratio [7].

Critical points in the finger skeleton are used as finger features for matching the finger vein images. Then the curve segments are extracted and tracing depend on this junction points [8].

This paper proposes an approach for finger vein recognition using three techniques for features extracting; Grey Level Cooccurrence Matrix (GLCM) [9], Tamura [10], and Scale Invariant Feature Transform (SIFT) [11].

This paper is organized as followed. Section 2 describes the database that has been used. Section 3 proposes the detail of our approach and verification system algorithm. Section 4 reports the experimental results that certify the validity of the proposed method. Finally, the conclusions of this paper are given in section 5 .

\section{DATABASE}

In this paper, Finger Vein USM (FV-USM) Database has been used as raw data in experimental results [12]. The Images in the database were collected from 123 Voluntaries comprising of 83 males and 40 females, who were staffs and students of University Sains Malaysia (USM). Every voluntary provided four fingers; left index, left middle, right index, right middle fingers resulting in a total of 492 fingers classed obtained. The resolution of captured images was 640 x 480 of gray level. Figure 1 gives a sample of FV-USM database.
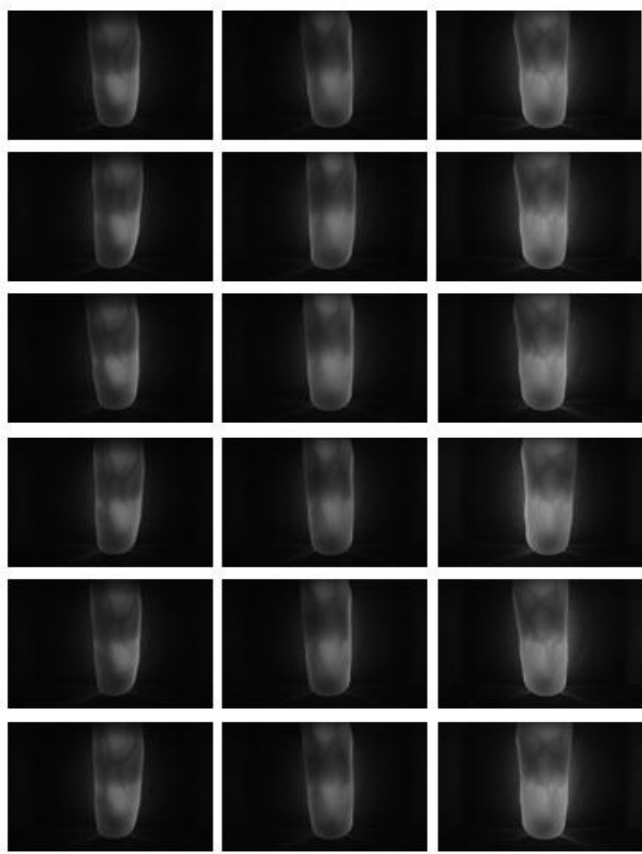

Fig 1: Samples of FV-USM database. 


\section{FINGER VEIN VERIFICATION SYSTEM}

The phase structure of the Finger vein verification system comprises of two stages: the training and the testing stages. Every stage has particular functions; all functions have explained in detail via the following subsections. The testing phase is the same of the training phase, but, in the testing phase, the features do not store in the database just entered to Minimum Distance Classifier (MDC) to recognize a person by choosing the person for which this distance is minimum. Figure 2 refers to the block diagrams of the training and testing stages of the proposed system.

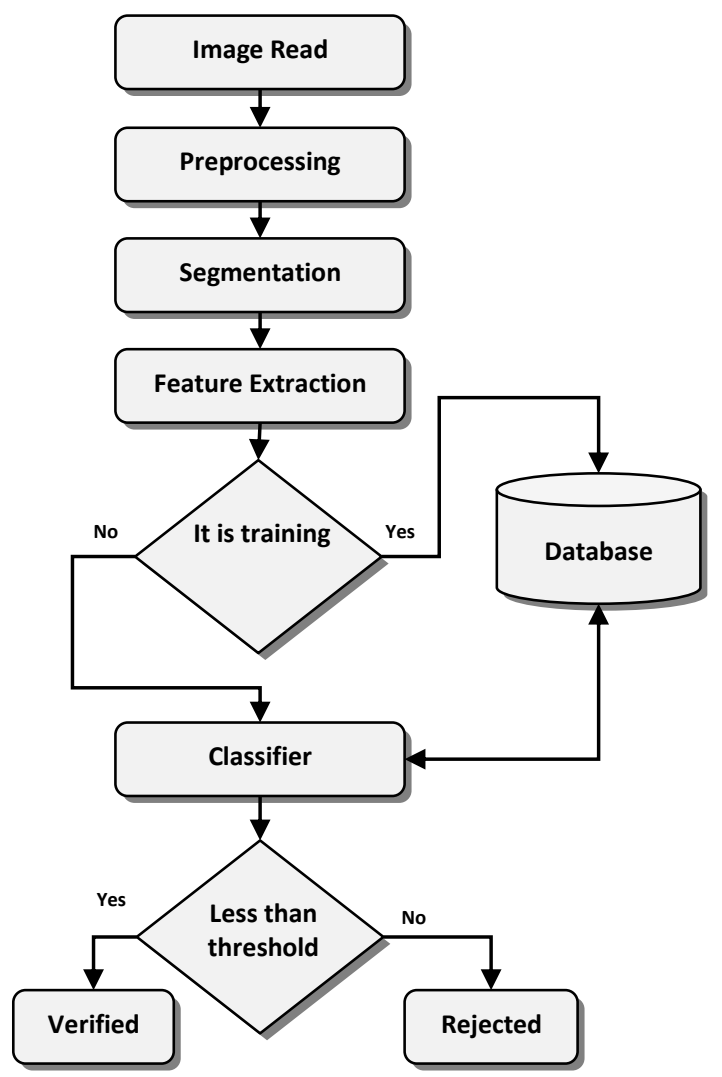

Fig 2: Block diagram of proposed verification system.

\subsection{Preprocessing}

According to the source of the database, finger vein images have been already converted into 256 gray level images. So, the next step is to enhance and suppress the undesired distortion of finger vein images. This has done by wavelet transforms [13]. These transforms smooth the image by blocking detailed information. The calculation of all input finger vein images once using a wavelet transform with Haar filter and to extract a vector of five features for each selected finger vein image, then an enhanced image has obtained with high clarity and free of noise.

\subsection{Segmentation}

Segmentation is an important procedure in the proposed finger vein system since the efficiency of the organization depends on the accuracy of the partitioning. Segmentation of the finger vein object from the background is a fundamental step in vein analysis. Most of the finger vein images contain a black background with significant noise. The finger vein images are segmented using mean's threshold segmentation, which is easy to carry out and exceedingly practiced. The result represents the region of interest (ROI) of finger vein that containing only the finger vein pattern to be recognized. Fig 3 epitomizes the result of implementing Mean's threshold on the source image.
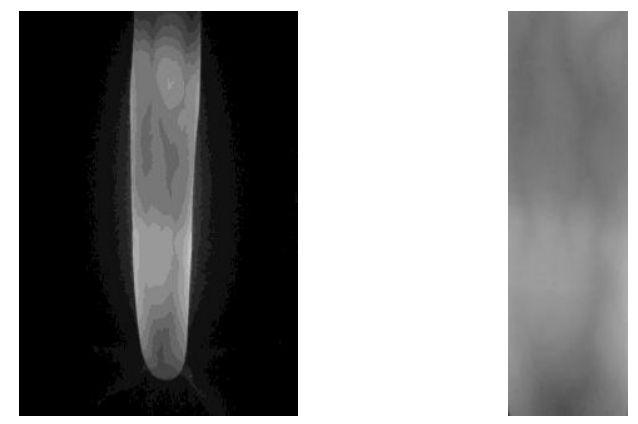

Fig 3: Result of segmentation method

\subsection{Feature Extraction}

Feature extraction is an important step for typical recognition systems, where the segmentation results have used as a guide to extract features. However, for each finger vein image, three methodologies of features extraction are used.

\subsubsection{Grey Level Co-occurrence Matrix}

Grey Level Co-occurrence Matrix (GLCM) has various features such as; contrast, correlation, energy, and homogeneity. All are computed from GLCM proposed in [9] for four directions $(0,45,90$, and135) to gray level image of finger vein. These features are described as below:

Contrast measures the amount of local variations in an image.

$$
\text { Contrast }=\sum_{i} \sum_{j}(\mathrm{i}-\mathrm{j})^{2} P(i, j)
$$

Correlation is a measurement of gray tone linear dependencies in the image.

$$
\text { Coreelatio }=\frac{\left(\sum_{i} \sum_{j}(\mathrm{i}-\mathrm{j})^{2} P(i, j)\right)-\mu_{x} \mu_{y}}{\sigma_{x} \sigma_{y}}
$$

Energy is a measurement of texture uniformity of an image, the more homogeneous the image, the larger the value.

$$
\text { Energy }=\sum_{i} \sum_{j} P(i, j)^{2}
$$

Homogeneity is a measurement of the amount of local uniformity present in the image

$$
\text { Homogeneity }=\sum_{i} \sum_{j} \frac{P(i, j)}{1+\left|i-j^{\prime}\right|}
$$

So, feature of ROI vein image have extracted by using four directions $\left(0^{\circ}, 45^{\circ}, 90^{\circ}\right.$, and $\left.135^{\circ}\right)$.

\subsubsection{Tamura}

Tamura [10] took the approach of devising texture features that correspond to human visual perception. They defined six textural features (coarseness, contrast, directionality, linelikeness, regularity and roughness) and compared them with psychological measurements for human subjects. The first three attained are very successful results and are used in our evaluation, both separately and as joint values.

Coarseness: Coarseness aims to identify the largest size at which a texture exists, even where a smaller micro texture exists. Computationally one first takes average at every point over neighborhoods the linear size of which are powers of 2 . 
The average A over the neighborhood of size $2 \mathrm{k} \times 2 \mathrm{k}$ at the point $(\mathrm{x}, \mathrm{y})$, where $\mathrm{k}=0,1, \ldots, 5$ is

$$
A_{k}(x, y)=\sum_{i=x-2^{k-1}}^{x+2_{j=y-}^{k-1}-2^{k-1}} \sum^{k-1} 2^{-1} f(i, j) / 2^{2 k}
$$

Then at each pixel, compute the difference $(\operatorname{Ek}(\mathrm{x}, \mathrm{y}))$ between pairs of non-overlapping moving averages in the horizontal and vertical directions. At each pixel, the value of $\mathrm{k}$ that maximizes $\operatorname{Ek}(\mathrm{x}, \mathrm{y})$ in either direction is used to set the best size (Sbest): Sbest $(x, y)=2 k$. The coarseness measure (Fcrs) is then computed by averaging Sbest $(\mathrm{x}, \mathrm{y})$ over the entire image.

Contrast: It aims to capture the dynamic range of grey levels in an image, together with the polarization of the distribution of black and white. The first is measured using the standard deviation of grey levels and the second the kurtosis $\alpha 4$. The contrast measure is therefore defined as:

$$
F_{\text {con }}=\frac{\sigma}{\left(\sigma_{4}\right)^{n}} \text { where, } \sigma_{4}=\frac{\mu_{4}}{\sigma^{4}}
$$

$\mu_{4}$ is the fourth moment about the mean and $\sigma$ is the variance. Experimentally, Tamura found $n=1 / 4$ to give the closest agreement to human measurements. This is the value we used in our experiments.

Directionality: Directionality is a global texture property. Patterns can be highly directional (e.g., a brick wall) or may be non-directional, as in the case of a picture of a cloud. The degree of directionality, measured on a scale of 0 to 1 , can be used as a descriptor. Thus, two patterns, which differ only in their orientation, are considered to have the same degree of directionality.

The texture features are coarseness, contrast and Directionality from Tamura method.

\subsubsection{Scale Invariant Feature Transform}

Scale Invariant Feature Transform algorithm involves four major stages for detection and description of local features, or key points, in the image [11]:

- Scale-space peak detection.

- Localization for Keypoints.

- Assignment for Orientation.

- Descriptor for each Keypoints.

Figure 4 briefs the main stages of SIFT-based approach.

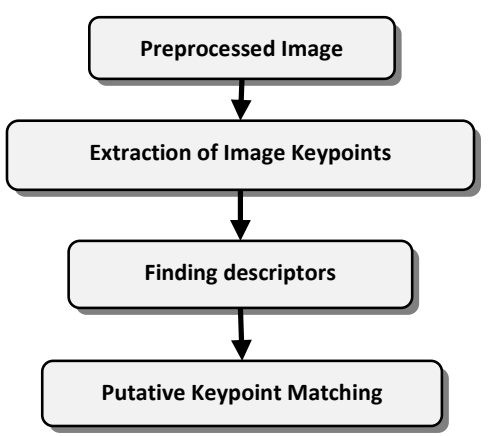

Fig 4: The main steps of SIFT based method

\subsubsection{Extraction Keypoints}

For finding possible identical finger veins in the database, RGB type of images should be converted to grayscale type by standard color space conversion for the image. The first process extracts of keypoints, then collects features. The locations that transfer determined information of the image contents are called keypoints. Keypoints can be identified by a feature vector where a collection of image statistics comes from local neighborhoods of the keypoint. The required keypoints and satisfactory features for extraction should be located in a distinguish site of the image. For any selected keypoints, a 128-dimentional features vector is produced by the histograms of local gradients of keypoint's neighborhoods.

\subsubsection{Putative Keypoint Matching}

Then, the best-bin-first method was used for matching the detected SIFT keypoints according to their feature vectors. A matching was performed for each keypoint (at location $x$ with feature $f$ ), with nearest neighbor (location $\tilde{x}$, feature vector $\tilde{f}$ ) correspondent one by measuring Euclidean distance.

\subsection{Classification}

The goal of this phase is testing a new input image with the trained images in the database. Classically, this phase separates into two manner; verification or identification. Verification is our goal in this work.

Block diagram of the verification mode is shown in Figure 5. The matching algorithm identifies similarity/dissimilarity between two given data groups. Many of these steps are implemented in this algorithm.

In verification mode, not only the biometric information is required from a user, but also the ID is required to be given. The matching stage gets in a single step by comparing the feature vector of the new image, and every image that has the same ID in the database. Then, pick up the image that gives the higher response. The Euclidean distance measure in this mode can be selected as a distance measure. Then, a threshold value is used to accept or reject the selected image.

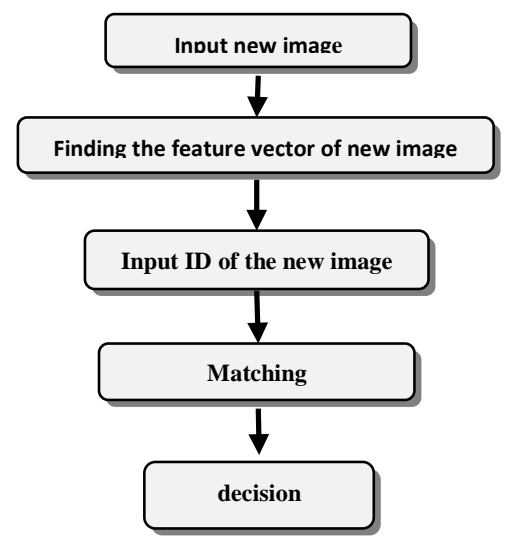

Fig 5: Block diagram of the verification mode

\section{EXPERIMENTAL RESULTS}

As mentioned previously the input data of the proposed system is a finger vein image. The finger vein verification system is trained and tested according to the system components. The results of these components are list below.

Four features (contrast, dissimilarity, homogeneity, energy) from GLCM as shown in Table 1 - Table 4. 
Table 1. Four features of GLCM at $(1,0 \circ)$

\begin{tabular}{|c|c|c|c|c|}
\hline Id & Contrast & Dissimilarity & Homogeneity & Energy \\
\hline 11 & 26.42005 & 0.418258 & 0.569077 & 0.023774 \\
\hline 12 & 26.49148 & 0.419197 & 0.569383 & 0.023863 \\
\hline 13 & 26.51523 & 0.429665 & 0.579535 & 0.023166 \\
\hline 14 & 26.41422 & 0.41508 & 0.562243 & 0.023169 \\
\hline
\end{tabular}

Table 2. Four features of GLCM at $\left(1,45^{\circ}\right)$

\begin{tabular}{|c|c|c|c|c|}
\hline Id & Contrast & Dissimilarity & Homogeneity & Energy \\
\hline 11 & 28.57206 & 0.321504 & 0.532555 & 0.021114 \\
\hline 12 & 28.61231 & 0.329104 & 0.533515 & 0.020761 \\
\hline 13 & 28.68794 & 0.334903 & 0.533481 & 0.021705 \\
\hline 14 & 28.67367 & 0.32627 & 0.529245 & 0.020133 \\
\hline
\end{tabular}

Table 3. Four features of GLCM at $(1,90 \circ)$

\begin{tabular}{|c|c|c|c|c|}
\hline Id & Contrast & Dissimilarity & Homogeneity & Energy \\
\hline 11 & 23.97794 & 0.396628 & 0.583682 & 0.023899 \\
\hline 12 & 23.77108 & 0.400526 & 0.584559 & 0.02381 \\
\hline 13 & 23.56985 & 0.407625 & 0.587054 & 0.024713 \\
\hline 14 & 23.73058 & 0.40406 & 0.582432 & 0.024339 \\
\hline
\end{tabular}

Table 4. Four features of GLCM at $\left(1,135^{\circ}\right)$

\begin{tabular}{|c|c|c|c|c|}
\hline Id & Contrast & Dissimilarity & Homogeneity & Energy \\
\hline $\mathbf{1 1}$ & 30.49208 & 0.318172 & 0.518954 & 0.027166 \\
\hline $\mathbf{1 2}$ & 30.105 & 0.316673 & 0.528253 & 0.027634 \\
\hline $\mathbf{1 3}$ & 29.35307 & 0.319503 & 0.527943 & 0.026496 \\
\hline $\mathbf{1 4}$ & 30.13669 & 0.320518 & 0.519713 & 0.02762 \\
\hline
\end{tabular}

Two features (coarseness, directionality) from Tamura as shown in Table 5.

Table 5. Two features of Tamura

\begin{tabular}{|c|c|c|}
\hline Id & Coarseness & Directionality \\
\hline 11 & 2.229008 & 43.1858 \\
\hline 12 & 2.229847 & 43.19385 \\
\hline 13 & 2.30998 & 43.1846 \\
\hline 14 & 2.230117 & 43.19225 \\
\hline
\end{tabular}

After combining overall feature vectors of GLCM, Tamura, and SIFT; the decision is reported.

The verification performance of our system will be measured by depending on the false accept rate (FAR) and false reject rate (FRR). Can calculating verification rate for our system through Total Success Rate (TSR) as follows:

$$
T S R=\left(1-\frac{F A R+F R R}{\text { Total }- \text { number }- \text { of }- \text { accesses }}\right) \times 100 \%
$$

The main factor that has an effect on the FAR and FRR is the automated Threshold. The selection of this value heavily depends on an application that we want the proposed system to work on. Therefore, the verification rate of the system has tested in different threshold values. The experiment performed in this section requires:

- Training set.

- Two testing sets:

- One for finding the FRR.

- Moreover, other for finding FAR.

The training set that is used in this experiments are 25 persons with five images for each one. In order to compute the FRR, images are not found in the training set but for the persons of the training set (i.e., known persons) must be tested. Therefore, the first testing set contains 25 persons with two images for each person. Therefore, the total number of genuine accesses is $50(25 \times 2)$.

To compute the FAR, images of unknown persons (i.e., persons outside the training set) must be tested.

Therefore, the second testing set contained 25 persons. With two images for each person. The total number of imposter accesses was $50(25 \times 2)$. Table 6 exhibits the results of the FRR, FAR, and TSR at different threshold values by using Euclidean distance at the matching stage.

From Table 6, one can say, "the verification accuracy of the system will increase when the verification threshold increases". One can use the values of FARs and FRRs to determine the best value of the threshold.

A threshold value equal to 0.9 because it gave a proximal balance between the FAR and FRR.

Table 6. The Verification Rate of Our System.

\begin{tabular}{|c|c|c|c|}
\hline $\begin{array}{c}\text { Threshold } \\
\text { value }\end{array}$ & FRR $(\boldsymbol{\%})$ & FAR $(\boldsymbol{\%})$ & TSR $(\%)$ \\
\hline 0.7 & 0.4 & 90.6 & $78.5 \%$ \\
\hline 0.75 & 3.6 & 80.6 & $79.2 \%$ \\
\hline 0.8 & 9 & 60 & $83.75 \%$ \\
\hline 0.85 & 11.2 & 39.3 & $88.37 \%$ \\
\hline 0.9 & 14 & 15 & 93.5 \\
\hline 0.95 & 19 & 3 & 96 \\
\hline
\end{tabular}

\section{CONCLUSION}

During scientific study, the conclusions which can be drawn from the proposed system are as follows: The finger vein image can be used as a recognizer between people. Automated segmentation using mean's and extracted ROI for the finger vein plays a central function in understanding image content for feature. Three set of feature extraction method are used for experimental results.

SIFT has been used for reducing unwanted (global features). The results totally are acceptable.

\section{REFERENCES}

[1] Shrikhande, Santosh P., and H. S. Fadewar. 2015 Finger vein recognition using Discrete Wavelet Packet Transform based features. In Advances in Computing, Communications and Informatics (ICACCI), 2015 International Conference on, pp. 1646-1651. IEEE.

[2] Kauba, Christof, and Andreas Uhl. 2015 Sensor ageing impact on finger-vein recognition. In Biometrics (ICB), 2015 International Conference on, pp. 113-120. IEEE.

[3] Nandhini, S., and D. Shyam. 2014 Real-Time FingerVein Recognition for Security and Monitoring System. In Applied Mechanics and Materials, vol. 550, pp. 194 203. Trans Tech Publications.

[4] Qin, Huafeng, Lan Qin, Lian Xue, Xiping He, Chengbo $\mathrm{Yu}$, and Xinyuan Liang. 2013 Finger-vein verification based on multi-features fusion. Sensors, 13, no. 11, pp, 15048-15067.

[5] Nguyen, Dat Tien, Young Ho Park, Kwang Yong Shin, Seung Yong Kwon, Hyeon Chang Lee, and Kang Ryoung Park. 2013 Fake finger-vein image detection 
based on fourier and wavelet transforms. Digital Signal Processing 23, no. 5 , pp. 1401-1413.

[6] Peng, J., Li, Q., El Latif, A. A. A., Wang, N., \& Niu, X. 2013 Finger vein recognition with gabor wavelets and local binary patterns. IEICE TRANSACTIONS on Information and Systems 96, no. 8, pp. 1886-1889.

[7] Mohan, Man, R. Prem Kumar, Rachit Agrawal, Surbhi Sharma, Malay Kishore Dutta, Carlos M. Travieso, and Jesus B. Alonso-Hernandez. 2015 Finger vein recognition using Integrated Responses of Texture features. In Bioinspired Intelligence (IWOBI), 2015 4th International Work Conference on, pp. 209-214. IEEE.

[8] Liu, Tong, Jianbin Xie, Wei Yan, Peiqin Li, and Huanzhang Lu. 2015 Finger-vein recognition with modified binary tree model. Neural Computing and Applications 26, no. 4, pp. 969-977.

[9] Eleyan, Alaa, and Hasan Demirel. 2011 Co-occurrence matrix and its statistical features as a new approach for face recognition. Turkish Journal of Electrical Engineering \& Computer Sciences 19, no. 1, pp. 97-107.

[10] Howarth, P. and Rüger, S. 2004 Evaluation of texture features for content-based image retrieval, International Conference on Image and Video Retrieval, July, pp.326334, Springer, Berlin Heidelberg.

[11] Lowe, David G. 2004 Distinctive image features from scale-invariant keypoints. International journal of computer vision 60, no. 2 ,pp. 91-110.

[12] Asaari, Mohd Shahrimie Mohd, Shahrel A. Suandi, and Bakhtiar Affendi Rosdi. 2014 Fusion of band limited phase only correlation and width centroid contour distance for finger based biometrics. Expert Systems with Applications 41, no.7, pp. 3367-3382.

[13] Elnasir, Selma, and Siti Mariyam Shamsuddin. 2014 Palm Vein Recognition based on 2D-Discrete Wavelet Transform and Linear Discrimination Analysis. Int. J. Advance Soft Compu. Appl 6, no. 3, pp. 43-59. 